\title{
Value of differential diagnosis of contrast-enhanced ultrasound in benign and malignant thyroid nodules with microcalcification
}

\author{
ZHIXIANG GAO, QIN LU and JIPING YAN \\ Department of Ultrasound, People's Hospital of Shanxi Province, Taiyuan, Shanxi 030012, P.R. China
}

Received October 12, 2018; Accepted January 28, 2019

DOI: 10.3892/ol.2019.10107

\begin{abstract}
Value of differential diagnosis of contrast-enhanced ultrasound in benign and malignant thyroid nodules with microcalcification was explored. A total of 184 patients with thyroid nodules with microcalcification, treated in People's Hospital of Shanxi Province from April 2015 to March 2017, were selected as research subjects. Contrast-enhanced ultrasound was used for imaging examination of the thyroid nodules. Three regions of interest were drawn at the positions with the strongest ultrasound imaging, for which the time-intensity curve (TIC), time to peak (Tp), peak intensity (Peak), area under curve (AUC) and mean transit time (MTT) were obtained separately. The features of contrast-enhanced ultrasound for malignant thyroid nodules were manifested as irregular focus edge, unclear boundary, low fiber reinforcement of the whole focus, uneven distribution of images and blood perfusion defect inside the focus, especially severe blood perfusion defect in the nodule center. The TIC showed a slow ascending and slow descending trend in general. The TIC features and the features of contrast-enhanced ultrasound for malignant thyroid nodules were prominently different from those for benign thyroid nodules. Compared with those in the surrounding normal tissues of thyroid gland, the Peak was remarkably shorter, and the AUC was notably smaller in the center and edge of malignant thyroid nodules $(\mathrm{P}<0.05)$; and the nodule center had obviously shorter Peak and smaller AUC than the nodule edge $(\mathrm{P}<0.05)$. Furthermore, in comparison with those of malignant thyroid nodules, the Peak was extended and AUC was enlarged markedly in the center and edge of benign thyroid nodules $(\mathrm{P}<0.05)$. In conclusion, the contrast-enhanced ultrasound can preferably compare the lesions of benign and malignant thyroid nodules with microcalcification, which possesses certain value in the differential diagnosis of benign and malignant thyroid nodules.
\end{abstract}

Correspondence to: Dr Jiping Yan, Department of Ultrasound, People's Hospital of Shanxi Province, 29 Shuangta Street, Taiyuan, Shanxi 030012, P.R. China

E-mail: yanjiping64@126.com

Key words: contrast-enhanced ultrasound, microcalcification, thyroid nodules, benign, malignant

\section{Introduction}

Calcification, a common pathological characteristic of thyroid diseases, occurs in the foci of $30 \%$ of benign thyroid nodules and $65 \%$ of malignant thyroid nodules (1). Calcifications in thyroid nodules can be classified as microcalcifications, coarse calcifications, annular calcifications and mixed calcifications, according to their size, but some studies have manifested that microcalcification presents the greatest differences between benign and malignant thyroid nodules $(2,3)$. Microcalcification can only be examined through high-resolution ultrasound due to its very small volume (maximum diameter of $2.0 \mathrm{~mm}$ ). Therefore, there are few reports on the diagnosis of benign and malignant thyroid nodules with microcalcification as the indicator. By virtue of infusing an ultrasound contrast agent into the blood circulation, contrast-enhanced ultrasound can enhance the differences in the ultrasonographic images between tissues and blood vessels, improve the resolution of ultrasonographic image results and increase the application range and depth of ultrasound (4). The development of contrast-enhanced ultrasound technique and its wide application in clinic make the accurate imaging examination of microcalcification in thyroid nodules possible. Therefore, in this study, contrast-enhanced ultrasound was applied to examine the microcalcification in benign and malignant thyroid nodules, and the differences in the quantitative parameters of contrast-enhanced ultrasound were compared, so as to explore the differential diagnosis value of contrast-enhanced ultrasound in benign and malignant thyroid nodules with microcalcification.

\section{Patients and methods}

Research subjects. A total of 184 patients with thyroid nodules accompanied by microcalcification, treated in People's Hospital of Shanxi Province (Taiyuan, China) from April 2015 to March 2017, were selected, including 100 males and 84 females. The age of patients ranged from 20 to 70 years, with an average age of $47.5 \pm 8.9$ years. The foci in thyroid nodules of all patients were examined via pathological sections, including 104 cases of benign thyroid nodules and 80 cases of malignant thyroid nodules. This research was reviewed and approved by the Ethics Committee of People's Hospital of Shanxi Province. All patients enrolled were aware of this research and each one and/or their guardians signed an informed consent. 
A

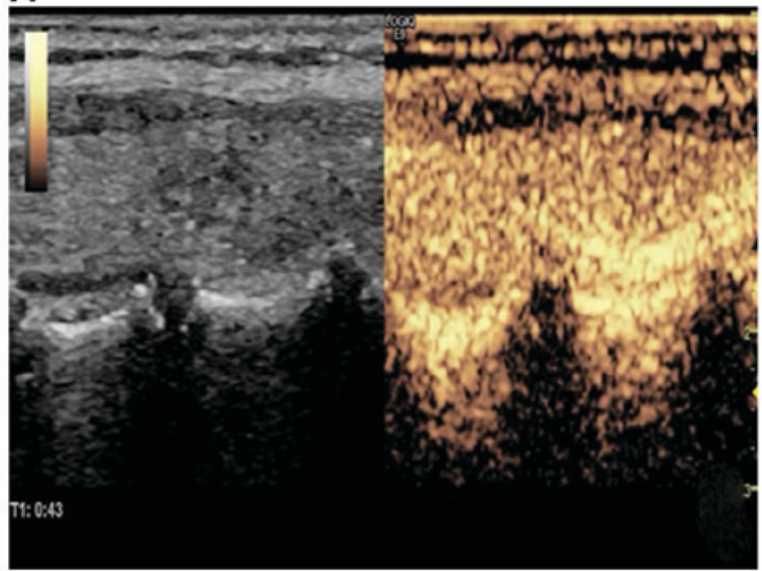

B

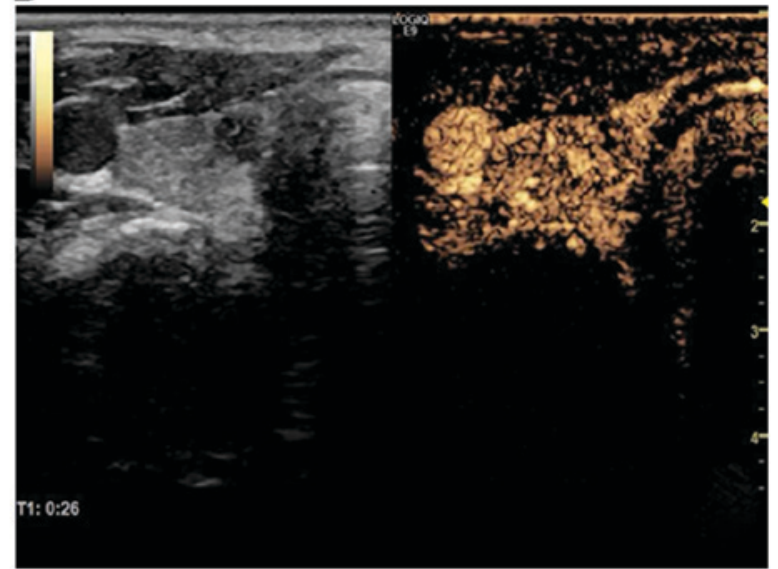

Figure 1. Comparison of contrast-enhanced ultrasound images of benign and malignant thyroid nodules with microcalcification. (A) Benign thyroid nodules. (B) Malignant thyroid nodules.
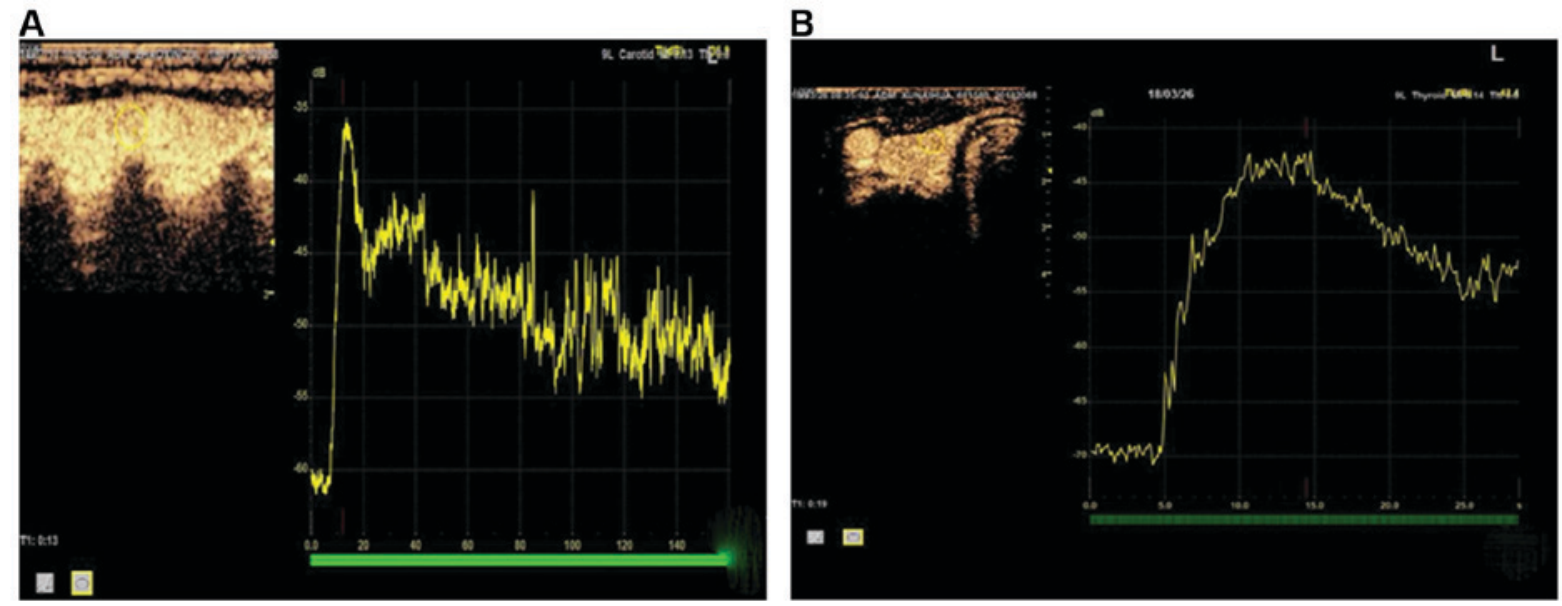

Figure 2. Comparison of TICs for benign and malignant thyroid nodules with microcalcification. (A) Benign thyroid nodules. (B) Malignant thyroid nodules. TIC, time-intensity curve.

\section{Methods}

Main reagents and instruments. Ultrasonic apparatus (Philips GmbH Healthcare, Hamburg, Germany), with built-in specialized analysis software for time-intensity curve (TIC), contrast-enhanced ultrasound-matching imaging techniques and variable frequency linear-array probe (frequency, 2-5 MHz) was utilized. Also, SonoVue contrast agent (Bracco SpA, Milan, Italy) composed of sulphur hexafluoride was used. During application, $2.4 \mathrm{ml}$ of freeze-dried powder of SonoVue contrast agent were dissolved in $5.0 \mathrm{ml}$ normal saline to prepare microbubble suspension.

Contrast-enhanced ultrasound examination for thyroid nodules. The research subjects were put in supine position, with their neck stretched sufficiently to expose the anterior region of the neck. Firstly, the conventional ultrasound was adopted to examine the thyroid nodules, so as to determine their characteristics, such as morphology, boundary and internal echo. Then, color Doppler ultrasound was applied to inspect the thyroid nodules and surrounding tissues, so as to determine the blood flow inside the foci and the surrounding tissues. The optimal sections for observation of the thyroid nodules were confirmed by integrating the examination findings of conventional ultrasound and color Doppler ultrasound. After that, the probe was fixed, and the contrast pulse sequence of the apparatus was switched on. Then, $2.4 \mathrm{ml}$ of prepared microbubble suspension of SonoVue contrast agent were rapidly injected into the blood vessels through the cubital vein, after which $5.0 \mathrm{ml}$ normal saline was injected immediately. Timer was started, and the data were collected while the contrast agent was injected. Moreover, constant and dynamic data $(120 \mathrm{sec})$ of every research subject were acquired. If the obtained contrast-enhanced images of the thyroid nodules were not satisfactory, the second injection of contrast agent through the cubital vein was performed for contrast-enhanced ultrasonography for the second time. The built-in data processing software of the ultrasonic apparatus was utilized to analyze the data collected. Three regions of interest were drawn at the positions with the strongest ultrasound imaging in the nodule center and edge, as well as the surrounding normal tissues of thyroid gland, for which the TIC, time to peak (Tp), peak intensity (Peak), area under curve (AUC) and mean transit time (MTT) were obtained separately. 
Statistical analysis.SPSS v.20.0 software (IBMCorp.,Armonk, NY, USA) was used for the statistical analysis of the collected data. Measurement data were expressed as mean \pm standard deviation. t-test was performed for the statistical analysis of the differences of the measurement data between two groups, and ANOVA was performed for the comparison of the measurement data between multiple groups. Least Significant Difference test was the post hoc test used. $\mathrm{P}<0.05$ was considered to indicate a statistically significant difference.

\section{Results}

Comparison of contrast-enhanced ultrasound images of benign and malignant thyroid nodules with microcalcification. The features of contrast-enhanced ultrasound images of benign and malignant thyroid nodules with microcalcification were compared. The results indicated that the foci of benign thyroid nodules had regular edges and clear boundaries and displayed equal or slightly high enhancement in general, without blood perfusion defect inside the foci. The malignant thyroid nodules were manifested as irregular focus edges, unclear boundaries and low fiber reinforcement of the whole foci, uneven distribution of images and blood perfusion defect inside the foci, especially severe blood perfusion defect in the nodule center (Fig. 1).

Comparison of TICs for benign and malignant thyroid nodules with microcalcification. The features of TICs for benign and malignant thyroid nodules with microcalcification were compared. The TIC for malignant thyroid nodules showed a slow ascending and slow descending trend in general: sluggish ascending section, smooth descending section, prolonged $\mathrm{Tp}$ and low and flat curve. The overall TIC for benign thyroid nodules was similar to that for surrounding normal tissues of thyroid gland, which was manifested as a rapid ascending and rapid descending trend: it rose and declined fast, with short Tp and narrow and high curve (Fig. 2).

Comparisons of quantitative parameters of TICs for benign and malignant thyroid nodules with microcalcification. The quantitative parameters of TICs for benign and malignant thyroid nodules with microcalcification were compared. According to the results, there were no significant differences in the Peak, Tp, AUC and MTT in the center and edge of benign thyroid nodules, as well as the surrounding normal tissues of thyroid gland $(\mathrm{P}>0.05)$. No significant differences in the $\mathrm{Tp}$ and MTT were presented in the center and edge of malignant thyroid nodules or in the surrounding normal tissues of thyroid gland $(\mathrm{P}>0.05)$. Compared with those in the surrounding normal tissues of thyroid gland, the Peak was remarkably shorter, and the AUC was notably smaller in the center and edge of malignant thyroid nodules $(\mathrm{P}<0.05)$; the nodule center had obviously shorter Peak and smaller AUC than the nodule edge $(\mathrm{P}<0.05)$. In addition, the Tp and MTT in the center and edge of benign thyroid nodules were not significantly different from those of malignant thyroid nodules $(\mathrm{P}>0.05)$. In comparison with those of malignant thyroid nodules, the Peak was extended and AUC was enlarged markedly in the center and edge of benign thyroid nodules $(\mathrm{P}<0.05$; Table I).

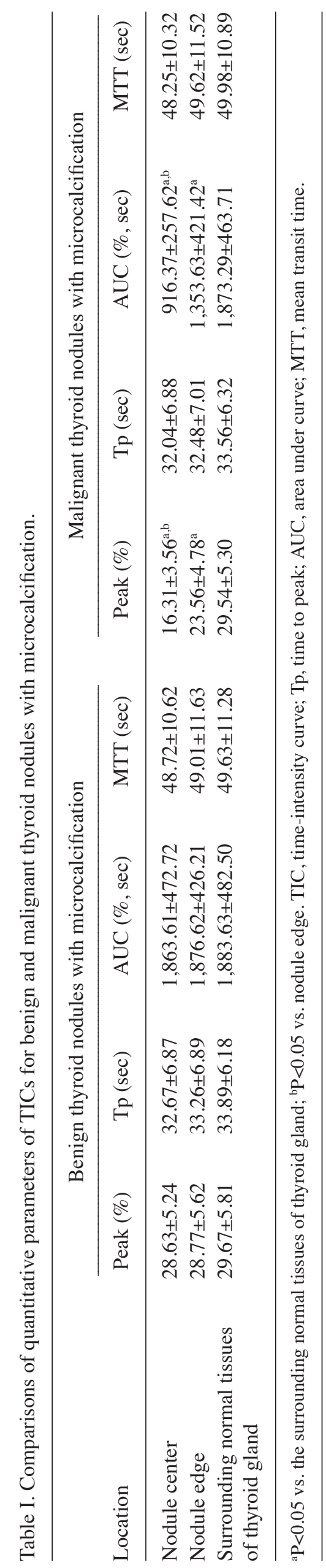




\section{Discussion}

Gravel-like microcalcification has attracted close attention in clinical practice. Studies have indicated that microcalcification possesses a fairly high incidence rate in papillary thyroid carcinoma, becoming a major morphological characteristic of the disease, while papillary thyroid carcinoma is a primary form of malignant lesion of the thyroid nodules $(5,6)$. In examinations, if the instruments are not sensitive enough, the pathologically puny foreign bodies of collagen and fibrosis, as well as a small amount of colloid, produce strong echoes in ultrasound, which may be confused with the echoes of true microcalcification, thus generating false-positive results. It has been reported that the thresholds of microcalcification diagnosed via ultrasound include maximum diameters $\leq 2.0$, $\leq 1.0$ and $\leq 0.5 \mathrm{~mm}$, of which the threshold of the maximum diameter $\leq 2.0 \mathrm{~mm}$ is used most extensively. The smaller the maximum diameter of microcalcification is, the higher the correlation with malignant thyroid nodules will be $(7,8)$. In consideration of the sensitivity of contrast-enhanced ultrasound examination, the maximum diameter of $2.0 \mathrm{~mm}$ was also selected as the threshold of microcalcification in this investigation. Major limitations exist in conventional ultrasound because the volume of early microcalcification is too small, and the sensitivity of conventional two-dimensional ultrasound is low. It is difficult to take the microcalcification as the basis for differential diagnosis of benign and malignant thyroid nodules. In contrast-enhanced ultrasound, the contrast between the blood vessels of foci and surrounding tissues is enhanced by perfusion of ultrasound contrast agent in the blood vessels of foci, thereby clearly reflecting the blood perfusion and vascular distribution inside the thyroid nodules $(9,10)$. The sensitivity of ultrasound in detecting the microcalcification can be improved significantly by virtue of contrast-enhanced ultrasound. Therefore, contrast-enhanced ultrasound was adopted to study the lesions of thyroid nodules with microcalcification.

In this study, the features of contrast-enhanced ultrasound for malignant thyroid nodules were manifested as irregular focus edge, unclear boundary, low fiber reinforcement of the whole focus, uneven distribution of images and blood perfusion defect inside the focus, especially severe blood perfusion defect in the nodule center. TIC showed a slow ascending and slow descending trend in general: sluggish ascending section, smooth descending section, prolonged $\mathrm{Tp}$ and low and flat curve. The TIC features and the features of contrast-enhanced ultrasound for malignant thyroid nodules were prominently different from those for benign thyroid nodules. Moreover, there were significant differences in the quantitative parameters of TICs for the center and edge of benign and malignant thyroid nodules. These results are related to the mechanism of microcalcification formation in the thyroid nodules and the pathological characteristics of benign and malignant lesions. The main component of microcalcification is calcium oxalate crystal (11). The major mechanism of microcalcification formation is as follows $(12,13)$ : i) thrombus is formed in the fibrovascular core of thyroid nodules, which develops into infarction, or necrosis of metastatic tumor cell nests in the lymph vessels occurs and triggers calcium deposit in dead tumor cells, thus forming gravel-like microcalcification. ii) The normal tumor cells in the thyroid nodules can release metabolic products and lead to dystrophic calcification of tumor cells, but such a calcification has no association with the apoptosis and necrosis of the tumor cells. iii) A large amount of bone morphogenetic protein-1 is expressed at the position of thyroid nodules, and the macrophages can produce osteopontin. In the malignant thyroid nodules with microcalcification, the existence of gravel-like calcium oxalate crystals can inhibit neovascularization inside the thyroid nodules and cause a lack of blood supply in the tumor (14). Furthermore, due to the very rapid cell proliferation of malignant thyroid tumors, the massive blood vessels inside the tumor have relatively small diameters and tortuous courses, which generate a great resistance to reduce the flow of blood and contrast agent toward the center of the nodules with microcalcification (15).

In conclusion, contrast-enhanced ultrasound can preferably compare the lesions of benign and malignant thyroid nodules with microcalcification, which possesses certain value in the differential diagnosis of benign and malignant thyroid nodules.

\section{Acknowledgements}

Not applicable.

\section{Funding}

No funding was received.

\section{Availability of data and materials}

The datasets used and/or analyzed during the present study are available from the corresponding author on reasonable request.

\section{Authors' contributions}

ZG and JY contributed to the contrast-enhanced ultrasound examination of thyroid nodules. QL and JY acquired, analyzed and interpreted the general data of patients. All authors read and approved the final manuscript.

\section{Ethics approval and consent to participate}

The study was approved by the Ethics Committee of the People's Hospital of Shanxi Province (Taiyuan, China). Signed informed consents were obtained from the patients and/or guardians.

\section{Patient consent for publication}

Not applicable.

\section{Competing interests}

The authors declare that they have no competing interests.

\section{References}

1. Lu Z, Mu Y, Zhu H, Luo Y, Kong Q, Dou J and Lu J: Clinical value of using ultrasound to assess calcification patterns in thyroid nodules. World J Surg 35: 122-127, 2011. 
2. Wang Z, Zhang H, Zhang P, He L and Dong W: Diagnostic value of ultrasound-detected calcification in thyroid nodules. Ann Acad Med Singapore 43: 102-106, 2014.

3. Jiang J, Shang X, Zhang H, Ma W, Xu Y, Zhou Q, Gao Y, Yu S and Qi Y: Correlation between maximum intensity and microvessel density for differentiation of malignant from benign thyroid nodules on contrast-enhanced sonography. J Ultrasound Med 33: 1257-1263, 2014.

4. Nemec U, Nemec SF, Novotny C, Weber M, Czerny C and Krestan CR: Quantitative evaluation of contrast-enhanced ultrasound after intravenous administration of a microbubble contrast agent for differentiation of benign and malignant thyroid nodules: Assessment of diagnostic accuracy. Eur Radiol 22: $1357-1365,2012$

5. Kim D, Kim DW, Heo YJ, Baek JW, Lee YJ, Park YM, Baek HJ and Jung SJ: Computed tomography features of benign and malignant calcified thyroid nodules: a single-center study. J Comput Assist Tomogr 41: 937-940, 2017.

6. Tugendsam C, Petz V, Buchinger W, Schmoll-Hauer B, Schenk IP, Rudolph K, Krebs M and Zettinig G: Ultrasound criteria for risk stratification of thyroid nodules in the previously iodine deficient area of Austria - a single centre, retrospective analysis. Thyroid Res 11: 3, 2018.

7. Ning CP, Ji QL, Fang SB, Wang HQ, Zhong YM and Niu HT: Distribution patterns of microcalcifications in suspected thyroid carcinoma: A classification method helpful for diagnosis. Eur Radiol 28: 2612-2619, 2018.

8. Koltin D, O'Gorman CS, Murphy A, Ngan B, Daneman A, Navarro OM, García C, Atenafu EG, Wasserman JD, Hamilton J, et al: Pediatric thyroid nodules: ultrasonographic characteristics and inter-observer variability in prediction of malignancy. $\mathrm{J}$ Pediatr Endocrinol Metab 29: 789-794, 2016.

9. Jiang J, Shang X, Wang H, Xu YB, Gao Y and Zhou Q: Diagnostic value of contrast-enhanced ultrasound in thyroid nodules with calcification. Kaohsiung J Med Sci 31: 138-144, 2015.
10. van Sloun RJG, Demi L, Postema AW, Jmch De La Rosette J, Wijkstra H and Mischi M: Entropy of ultrasound-contrast-agent velocity fields for angiogenesis imaging in prostate cancer. IEEE Trans Med Imaging 36: 826-837, 2017.

11. Na DG, Kim DS, Kim SJ, Ryoo JW and Jung SL: Thyroid nodules with isolated macrocalcification: Malignancy risk and diagnostic efficacy of fine-needle aspiration and core needle biopsy. Ultrasonography 35: 212-219, 2016.

12. Özemir IA, Bayraktar B, Anılır E, Orhun K, Eren T, Sağıroğlu J, Ceyran $A B$, Yiğitbaşı $R$ and Alimoğlu $O$ : The association of papillary thyroid cancer with microcalcification in thyroidnodules with indeterminate cytology based on fine-needle aspiration biopsy. Turk J Med Sci 46: 1719-1723, 2016.

13. Majstorov V: Ultrasonographic findings in patients with benign and malignant thyroid nodules who underwent ultrasound guided fine needle aspiration cytology. Open Access Maced J Med Sci 3: 689-693, 2015 .

14. Khoo ML, Asa SL, Witterick IJ and Freeman JL: Thyroid calcification and its association with thyroid carcinoma. Head Neck 24: 651-655, 2002.

15. Liu MJ, Men YM,Zhang YL, Zhang YX and Liu H: Improvement of diagnostic efficiency in distinguishing the benign and malignant thyroid nodules via conventional ultrasound combined with ultrasound contrast and elastography. Oncol Lett 14: 867-871, 2017.

This work is licensed under a Creative Commons Attribution-NonCommercial-NoDerivatives 4.0 International (CC BY-NC-ND 4.0) License. 